Louisiana State University

LSU Digital Commons

Faculty Publications

Department of Chemistry

6-7-2018

\title{
Dynamics of Phospholipid Membranes beyond Thermal Undulations
}

\author{
Sudipta Gupta \\ Louisiana State University \\ Judith U. De Mel \\ Louisiana State University \\ Rasangi M. Perera \\ Louisiana State University \\ Piotr Zolnierczuk \\ Spallation Neutron Source \\ Markus Bleuel \\ NIST Center for Neutron Research
}

See next page for additional authors

Follow this and additional works at: https://digitalcommons.Isu.edu/chemistry_pubs

\section{Recommended Citation}

Gupta, S., De Mel, J., Perera, R., Zolnierczuk, P., Bleuel, M., Faraone, A., \& Schneider, G. (2018). Dynamics of Phospholipid Membranes beyond Thermal Undulations. Journal of Physical Chemistry Letters, 9 (11), 2956-2960. https://doi.org/10.1021/acs.jpclett.8b01008

This Article is brought to you for free and open access by the Department of Chemistry at LSU Digital Commons. It has been accepted for inclusion in Faculty Publications by an authorized administrator of LSU Digital Commons. For more information, please contact ir@lsu.edu. 


\section{Authors}

Sudipta Gupta, Judith U. De Mel, Rasangi M. Perera, Piotr Zolnierczuk, Markus Bleuel, Antonio Faraone, and Gerald J. Schneider 


\title{
Dynamics of Phospholipid Membranes beyond Thermal Undulations
}

\author{
Sudipta Gupta, ${ }^{1}$ Judith U. De Mel, ${ }^{1}$ Rasangi M. Perera, ${ }^{1}$ Piotr Zolnierczuk, ${ }^{2}$ Markus Bleuel, ${ }^{3}$ \\ Antonio Faraone, ${ }^{3}$ and Gerald J. Schneider ${ }^{1,4}$ \\ ${ }^{1}$ Department of Chemistry, Louisiana State University, Baton Rouge, LA 70803, USA \\ ${ }^{2}$ Jülich Centre for Neutron Science (JCNS), Outstation at SNS, POB 2008, 1 Bethel Valley Road, \\ TN 37831, Oak Ridge, USA \\ ${ }^{3}$ NIST Center for Neutron Research, National Institute of Standards and Technology, \\ Gaithersburg, Maryland 20899-8562, USA \\ ${ }^{4}$ Department of Physics \& Astronomy, Louisiana State University, Baton Rouge, LA 70803, \\ $U S A$
}

\begin{abstract}
We investigated the molecular dynamics of unilamellar liposomes by neutron spin echo spectroscopy. We report the first experimental evidence of a short-range motion at the lengthscale of the size of the head group of a lipid. The associated mean squared displacement shows a $t^{0.26}$ dependence in the pico-to nanosecond region that indicates another process beyond the predictions of the Zilman-Granek model $\left(t^{0.66}\right)$ and translational diffusion $\left(t^{1}\right)$. A comparison with theory shows that the observed low exponent is associated with a non-Gaussian transient trapping of lipid molecules in a local area and supports the continuous time random walk model. The analysis of the mean squared displacement leads to the important conclusion that the friction at the interface between water and liposomes plays a minor role. Center of mass diffusion of
\end{abstract}


liposomes and transient trapping of lipids define the range in which the ZG model can be applied to analyze membrane fluctuations.

Phospholipids are an integral part of cell membranes in living cells and are ubiquitous in nature. Following its widespread applications in agriculture, food processing and pharmaceuticals, deeper understanding of the structure and dynamics of such membranes is essential. ${ }^{1}$ Phospholipids with hydrophilic head and hydrophobic tail, spontaneously self-assemble in aqueous media to form liposomes. The lipid bilayers appear to be a 3D continuous closed vesicle where the shape fluctuations in nanosecond time scale depend on the bending modulus of the membrane. ${ }^{2-3}$ The bending mechanism is related to important biological functioning like cellular uptake and release. ${ }^{4}$ Considering an ensemble of membrane plaquettes at random orientations, Zilman and Granek were successful in estimating the bending stiffness to describe the thermal membrane undulations.

However, the closest packing of lipid molecules causes a viscoelastic and heterogeneous behavior, which leads to random anomalous diffusion observed in living cell membranes and their molecular dynamics is far from being understood. ${ }^{5-8}$ The origin of such anomalous motions is controversial. Different models have been proposed, which try to identify the most biologically relevant mechanisms, like the continuous time random walk (CTRW) models that assume transient trapping of lipids ${ }^{9}$ and fractional Brownian motion (FBM) models that account for the geometrical inhomogeneities in lipid bilayers. ${ }^{10}$ Such motions in the bilayers are supposed to be coupled to the overall vesicle dynamics at appropriate length and time scales, and a proper exploration of the 
anomalous motions in lipid bilayer is crucial to understand diffusion-limited reactions, signaling and regulatory process in bio macromolecules. ${ }^{11-12}$

Neutron spin echo (NSE) spectroscopy has proved to be a powerful tool to follow the molecular motions in liposomes. ${ }^{2-3,13}$ In this communication, we use NSE to explore the molecular processes in lipid bilayers. As a major result of our study, we find evidence for local trapping and nonGaussianity in the motion of lipids at the time-scale from picoseconds to nanoseconds, which strongly supports CTRW models. Additionally, we identify the time region between the fast-local trapping and the slower center of mass diffusion of liposomes in which the ZG model accurately describes the experimental data and reveal that the energy dissipation between the membranes and the solvent plays a lesser role in case of liposomes than in case of objects with a lower bending modulus and single interfaces like in microemulsions.

We studied the dynamics of dioleoyl-phosphocholine (DOPC) at $20^{\circ} \mathrm{C}$ and compare the results with those of other phospholipids, like, distearoyl-phosphocholine (DSPC) at $65^{\circ} \mathrm{C}$, dimyristoyl-phosphocholine (DMPC) at $37^{\circ} \mathrm{C}$, and L- $\alpha$-phosphatidylcholine (SoyPC) at $30^{\circ} \mathrm{C}$. These different temperatures are necessary to compare all of the liposomes in their respective fluid-like phases.

We used small-angle neutron scattering (SANS) and cryo-transmission electron microscopy (cryoTEM) to characterize the static structure. NSE and dynamics light scattering (DLS) were used to study dynamics. A more detailed descripted is omitted here, but can be found in the ESI. 
A simple approach to analyze the intermediate scattering function, $S(Q, t)$, as determined by NSE experiments, uses the Zilman-Granek (ZG) model that introduces the bending rigidity to describe the membrane dynamics ${ }^{14}$

$$
\frac{S(Q, t)}{S(Q)}=\exp \left[-\left(\Gamma_{Q} t\right)^{2 / 3}\right] .
$$

The only free parameter is the $Q$-dependent decay rate, $\Gamma_{Q}$, from which we derive the intrinsic bending modulus, $\kappa_{\eta}$, by ${ }^{13,15-16}$

$$
\frac{\Gamma_{q}}{Q^{3}}=0.0069 \gamma \frac{k_{B} T}{\eta} \sqrt{\frac{k_{B} T}{\kappa_{\eta}}}
$$

Here $\eta$ is the viscosity, $k_{B}$ the Boltzmann constant, $T$ the temperature, and $\gamma$ is a weak, monotonously increasing function of $\kappa_{\eta} / k_{B} T .{ }^{14}$ In case of lipid bilayers usually $\kappa_{\eta} / k_{B} T \gg 1$, leading to $\gamma=1 .^{2,13-14,16-17}$ Equation 2 can be derived from a modified ZG theory that includes intermonolayer friction. A detailed discussion is omitted here, but can be found in the literature. ${ }^{13,15-16}$

In addition, the contribution of the translational center of mass diffusion, $D_{t}$, of the liposomes needs to be included in the analysis of the dynamic structure factor, and we rewrite equation 1 13,18

$$
\frac{S(Q, t)}{S(Q)}=\exp \left(-D_{t} Q^{2} t\right) \exp \left[-\left(\Gamma_{q} t\right)^{2 / 3}\right]
$$

by assuming ZG motion and center of mass diffusion are statistically independent. The separate measurement of $D_{t}$ by DLS avoids additional free parameters. 
Figure 1(a) displays $\mathrm{S}(Q, t)$ by DLS on DOPC. The corresponding hydrodynamic radius was found to be $R h=664 \pm 20 \AA$ at a lipid mass fraction $\phi_{w}=0.25 \%$. The size distribution is presented in the inset with the corresponding log-normal polydispersity of $38 \pm 2 \%$. Figure 1 (b) shows SANS data for $\phi_{w}=0.25 \%$ and $5 \%$ samples. After dividing the intensities by the respective volume fractions, the intensity values at the two different concentrations are virtually identical, particularly in the Q range where we studied the dynamic correlation function by NSE (highlighted area). A more detailed inspection of the SANS data by a coreshell model adapted for liposomes, ${ }^{19}$ yields a radius of $536 \pm 2 \AA$ and a bilayer thickness of 36 $\pm 1 \AA$ A. Using a Schulz distribution or a log-normal distribution for the radius polydispersity yields $30 \pm 2 \%$. Cryo-TEM suggests a radius of $\sim 550 \AA$, cf. inset of Fig. 1 (b). Within the experimental uncertainties the values of the diameters are consistent and agree very well with those reported in the recent literature. ${ }^{13}$
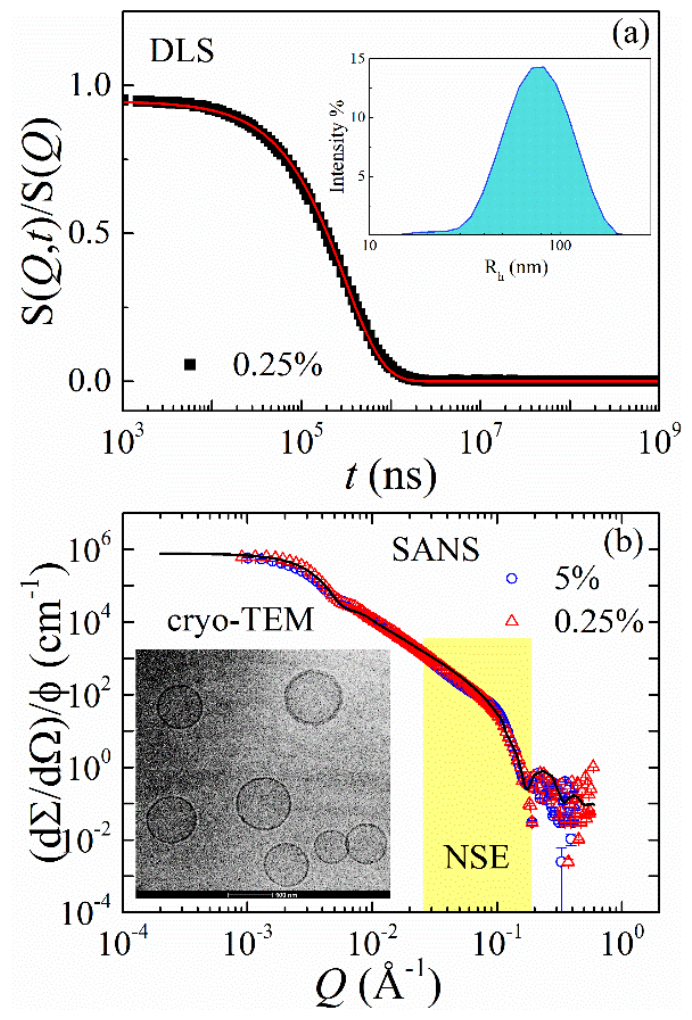
Figure 1. (a) The dynamic structure factor, $S(Q, t) / S(Q)$, from $D L S$ autocorrelation function as a function of time. (b) SANS scattering intensity for, $\phi_{w}=5 \%$ and $0.25 \%$ DOPC dispersed in $\mathrm{D}_{2} \mathrm{O}$. The solid line represents the vesicle form factor. Inset: Cryo-TEM image.

Our SANS analysis yields the total number of lipids per liposome, $n_{L}=9.9 \pm 0.2 \times 10^{4}$. This number corresponds to an average area per lipid of $a=69 \pm 2 \AA^{2}$, which matches the value reported in literature..$^{20}$
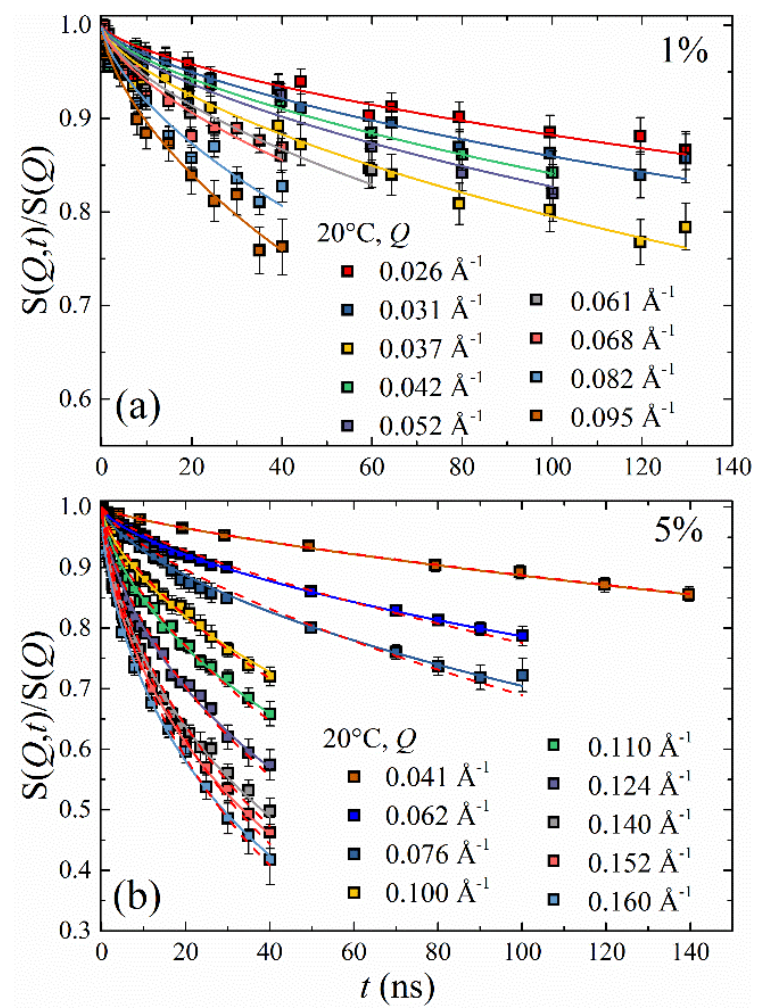

Figure 2. Dynamic structure factor, $S(Q, t) / S(Q)$, as a function of Fourier time, $t$, for different $Q$ 's as indicated for (a) 1\%, and (b) 5\% samples. The solid (-) and the dashed (---) lines represent the fits using equation 1 and 3, respectively. 
Figure 2 (a) and (b) illustrate $S(Q, t)$ by NSE in the fluid phase for $\phi_{w}=1 \%$ and $5 \%$ DOPC samples. Using equation 1 and $\eta=\eta_{D_{2} O}$ we obtain the solid lines, and $\kappa_{\eta, 1} /\left(k_{B} T\right)=20 \pm 3$ and $20 \pm 2$, for $\phi_{w}=1$ and $5 \%$, respectively. Stimulated by earlier work, ${ }^{13,18}$ we test the consequence of taking into account a finite diffusion coefficient, separately determined from DLS. The fits by equation 3 (dashed lines in figure 2) can hardly be distinguished from the previous case and yield $\kappa_{\eta, 2} /\left(k_{B} T\right)=53 \pm 5$ and $44 \pm 3>\kappa_{\eta, 1} /\left(k_{B} T\right)$. These $\kappa_{\eta, 2} /\left(k_{B} T\right)$ disagree with typical values $\kappa_{\eta} \approx 20 k_{B} T$, reported for large unilamellar vesicles (LUVs), DOPC and other phospholipids. ${ }^{2,21-23}$ To identify the source of this contradiction, we now consider the local dissipation of the membrane fluctuation energy at the membrane solvent interface, similarly to the analysis of NSE on microemulsions. ${ }^{24}$ Therefore, we used the effective solution viscosity, $\eta=\eta_{\mathrm{D} 20+\text { liposome }}$, in equation 2 , and recalculated $\kappa_{\eta}$. This leads to $\kappa_{\eta, 3} /\left(k_{B} T\right)=9 \pm 1\left(1\right.$ and $5 \%$ ), for $\eta=\eta_{D_{2} O}$ (in Eq. 2), and $\kappa_{\eta, 4} /\left(k_{B} T\right)=22 \pm 3$ and $19 \pm 2$, for 1 and $5 \%$ respectively, for $\eta=\eta_{\mathrm{D}_{2} \mathrm{O}+\text { liposome. }}$. While one value appears to be too low, the other seems to reflect the literature. Hence, the analysis appears to be ambiguous and deserves further attention. This becomes even more obvious if we vary the diffusion coefficient in our analysis, up to higher values found by PFG-NMR, cf. figure $3 .{ }^{25} \mathrm{We}$ also included the value of $\kappa_{\eta}$ for the $0.1 \%$ sample reported from the literature. ${ }^{13}$ The horizontal line represents $20 k_{\mathrm{B}} T$.

The apparent saturation and high uncertainty for $D_{\mathrm{t}} \geq 0.6 \AA^{2} \mathrm{~ns}^{-1}$ is due to the fact that the model fails to describe $S(Q, t)$ for large $D_{\mathrm{t}}$ (highlighted), cf. ESI. Figure 3 suggests a general trend depicting an exponential increase into unphysically large values of $\kappa_{\eta}$. The $0.1 \%$ sample 
is not affected, because in this case $\eta_{\mathrm{D}_{2} \mathrm{O}+\text { liposome }} \approx \eta_{\mathrm{D}_{2} \mathrm{O}}$ and there the measured solution viscosity is identical to the solvent viscosity.

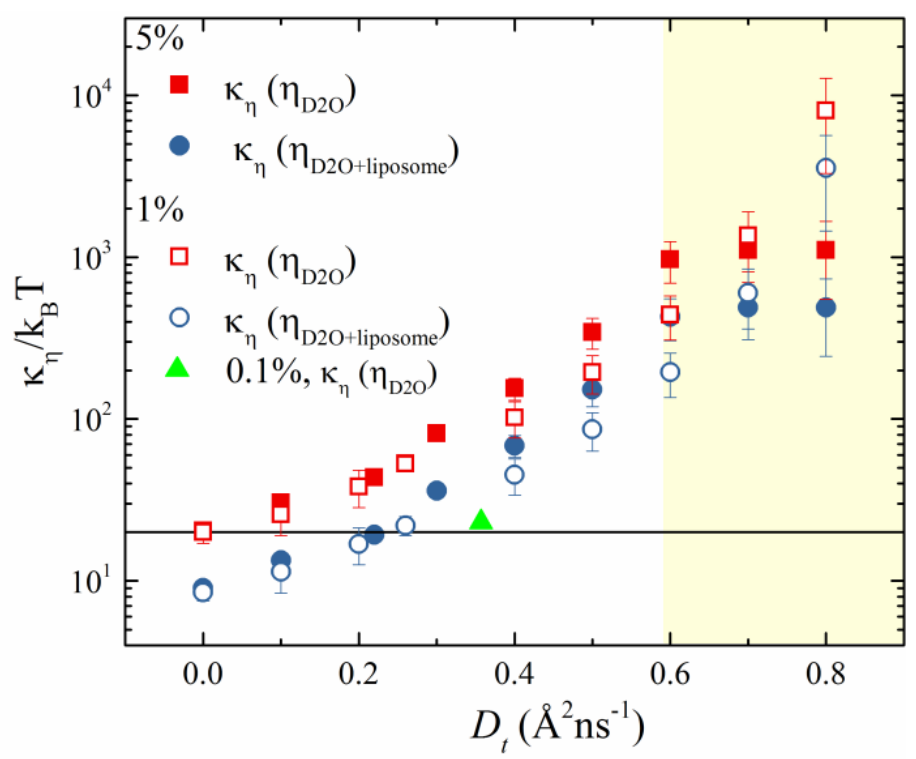

Figure 3. The intrinsic bending modulus, $\kappa_{\eta} /\left(k_{B} T\right)$, calculated for different translational diffusion, $D_{t}$, using the solvent viscosity, $\eta_{D_{2} O}$, or the measured solution viscosity, $\eta_{\mathrm{D}_{2} \mathrm{O}+\text { liposome, }}$ for the viscosity, $\eta$, used in equation 2.

To resolve this issue, hereafter, we calculate the mean square displacement $\left(\left\langle\Delta r(t)^{2}\right\rangle\right.$, MSD) from $S(Q, t)$ and find a correct and non-ambiguous answer that $\eta_{\mathrm{D}_{2} \mathrm{O}}$ is correct, as has been previously used by numerous studies. ${ }^{26-27}$ In addition, we reveal another process that can be associated with non-Gaussian transient trapping of lipid molecules, predicted by molecular dynamics simulations at very short times. ${ }^{28}$ Both diffusion and transient trapping define the range in which the $Z G$ model can be used to analyze membrane fluctuations. 
We used a cumulant expansion to obtain $\left\langle\Delta r(t)^{2}\right\rangle$ and the non-Gaussianity parameter, $\alpha_{2}(t)=$ $\frac{d}{d+2} \frac{\left\langle\Delta r(t)^{4}\right\rangle}{\left\langle\Delta r(t)^{2}\right\rangle^{2}}-1$, which is defined by the quotient of the fourth $\left\langle\Delta r(t)^{4}\right\rangle$ and the second moment squared $\left\langle\Delta r(t)^{2}\right\rangle^{2}$ and $d=3$, the dimension in space, cf. figure $4 .{ }^{26,29}$
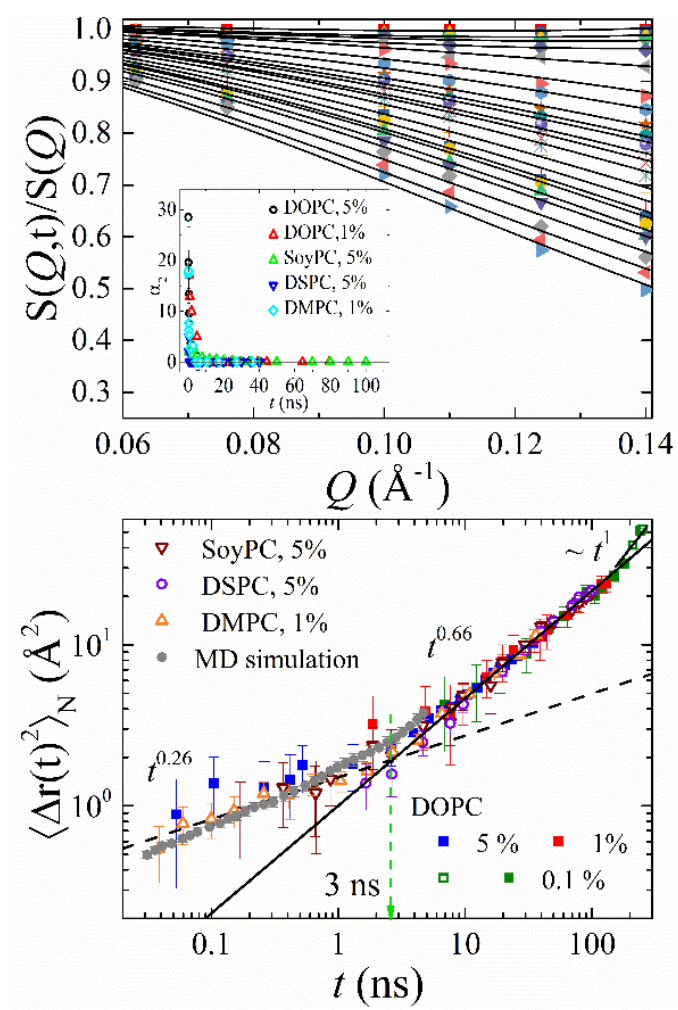

Figure 4. (a) Dynamic structure factor, $S(Q, t)$, as a function of $Q$ for $5 \%$ DOPC, at $20{ }^{\circ} \mathrm{C}$ in $\mathrm{D}_{2} \mathrm{O}$ for different Fourier times ranging from 0.05 to $40 \mathrm{~ns}$ (top to bottom) (Inset) The nonGaussian parameter for DOPC, DSPC, DMPC and SoyPC samples. (b) The normalized mean square displacement $\left\langle\Delta r(t)^{2}\right\rangle_{N}$ vs. Fourier time $t$, calculated for $0.1 \%, 1 \%$ and $5 \%$ DOPC, 10\% DSPC, $1 \%$ DMPC and 5\% SoyPC samples. The data for $0.1 \%$ DOPC and 1\% DMPC are calculated using $S(Q, t) / S(Q)$ from the literature. ${ }^{13,30}$ The solid and dashed lines represent the experimental power-law dependence, filled circles from MD simulation. ${ }^{28}$ 
Figure 4 (a) exemplifies the extraction of $\left\langle\Delta r(t)^{2}\right\rangle$ from the normalized dynamic structure factor, $S(Q, t) / S(Q)$ of DOPC with $\phi_{w}=5 \%$. The inset reports a Gaussian behavior for $t>3 \mathrm{~ns}$, but a non-Gaussian behavior for $t<3 \mathrm{~ns}$. For the sake of a better comparability, we plotted the MSD normalized to the ZG contribution. The influence of the temperature can be found in the ESI. We added different concentrations and different phospholipids to accomplish a more comprehensive picture, including 0.1\% DOPC, ${ }^{13} 1 \%$ DMPC, ${ }^{30} 5 \%$ DSPC, and 5\% SoyPC, all in the fluid phase.

In figure 4 we identify three regions that can be distinguished by different power laws $\left(\approx t^{n}\right)$. While the center of mass diffusion $\left(t^{1}\right)$ is almost outside the observation window (open squares), the intermediate region exhibits $\sim t^{0.66 \pm 0.01}$, and at low Fourier times $(\mathrm{t}<3 \mathrm{~ns})$, we find $t^{0.26 \pm 0.03}$. The time window, $3 \leq t \leq 180 \mathrm{~ns}$, for the $t^{0.66 \pm 0.01}$ region seems to be independent from the concentration and from the chemical composition of the lipids. We observe that the contribution of the center of mass diffusion of the liposomes shifts out of the observation window of the NSE for higher concentrations.

Flenner at al. ${ }^{31}$ observed very rich dynamics in phospholipids, a ballistic motion, indicated by the $t^{2}$, at the fast time scale, and a subdiffusive $t^{0.67}$ (whole lipid) or $t^{0.43}$ (carbons in the tail) at the intermediate time scale. The motion in the intermediate time range corresponds to the socalled thermal undulations defined by ZG model, and the anomalous diffusion predicted by Monte Carlo simulations. ${ }^{14,32}$ At the slow time scale, Flenner et al. ${ }^{31}$ report a $t^{1}$ behavior, which associated with the diffusion of lipids. 
To associate it to our observations, let's compile some of the relevant facts, to discuss the lower exponent $\left(t^{0.26 \pm 0.03}\right)$ for $\mathrm{t} \lesssim 3 \mathrm{~ns}$ : (i) The length-scale associated with our observation window is, $6 \AA \sqrt{\left\langle\Delta r(t)^{2}\right\rangle} \lesssim 14 \AA$, for 20 ps $\lesssim \mathrm{t} \lesssim 3$ ns. (ii) The dynamics becomes nonGaussian for $\mathrm{t}<3$ ns and $\alpha_{2}$ increases with decreasing time. (iii) In agreement with the values reported in literature, we determined the thickness of the bilayer, $t_{\mathrm{b}} \approx 36 \AA \mathrm{.}^{2,33}$ The thickness of the bilayer in concert with the MSD shows that the corresponding dynamics occurs at the length-scale of single lipids and is faster than the anomalous diffusion in the ZG regime. (iv) The area per lipid is $69 \pm 2 \AA^{2}$ for DOPC, which leads to an estimate of the perimeter radius $\approx 5 \AA \lesssim \sqrt{\left\langle\Delta r^{2}(3 \mathrm{~ns})\right\rangle}$. Therefore, the motion is very local, which excludes a (hindered) diffusive motion of the liposomes at this time and length-scale. This also excludes the by far slower flip-flop, rotational and lateral diffusion mechanisms. ${ }^{34}$ (v) Two theoretical models are available to account for an exponent that is on the order of 0.3: (a) Fractional Brownian motion (FBM), which is an expansion of the Brownian diffusion. A so-called anticorrelation causes the exponent of $\sim 0.3$ within the FBM model.${ }^{28}$ However, this model is incompatible with the observed non-Gaussian behavior. ${ }^{9,} 28$ (b) The continuous time random walk (CTRW) model assumes a random walk like motion, but with random trapping times. It predicts sub-diffusion and non-Gaussianity. ${ }^{9}{ }^{28}$ Our experimental results favor therefore the CTRW model. (vi) Molecular dynamics (MD) simulations of a palmitoyl-oleoyl-phosphatidylethanolamine (POPE) lipid bilayer suggest a fluctuation like motion, including a finite non-Gaussianity for $t<3 \mathrm{~ns}$, associated with the transition from a $t^{0.7}$ to $t^{0.33 \pm 0.02}$ power law at $\mathrm{t} \approx 3 \mathrm{~ns}$ with decreasing time.$^{28}$ Our experimental observations seem to be independent of the specific lipid. Therefore, we include the results of MD simulations in figure 4 and find a surprisingly good agreement. The MD simulations show that lipids are caught in an area $\lesssim 7 \AA \times 7 \AA$, even at a 
longer time of up to $20 \mathrm{~ns} .{ }^{28}$ In the experiments the contribution of the membrane undulations to the MSD becomes stronger for $\mathrm{t}>3 \mathrm{~ns}$ and hides that of the lateral lipid motion. Thus, the simulations are well compatible with the experiments. From the simulations, we know that the low exponent is a consequence of the divergence of the mean trapping time, which naturally involves a non-Gaussian behavior. In other words, we identified a new region which corresponds to a lateral motion of lipids, which are trapped within a certain range that is on the order of the size of the molecule. The MD simulations and mode-coupling theory calculations by Flenner et al. ${ }^{31}$ suggest that our experimental observation of a trapped motion is associated with the motion carbon tail of the fatty acid.

Our observations have important consequences. (i) The analysis of the ZG membrane undulation is only valid in a certain time range. In our case, it is constrained by the transient trapping of the lipids on the one side, and by the center of mass diffusion of the liposomes on the other side. (ii) Depending on the time range of the experiment, the center of mass diffusion can substantially change $S(Q, t)$. (iii) The MSD tells us whether the diffusion needs to be included in the analysis. Therefore, we can analyze the data accordingly, and find that only the case $\eta=\eta_{D_{2} O}$ yields a correct description of the experimental data, which justifies the procedure that has been used in numerous cases. ${ }^{2-3,16,30}$ (iv) Item (iii) implies that the local dissipation of the membrane fluctuation energy at the interface with the surrounding solvent plays a lesser role than in microemulsions.

\section{Acknowledgement}


The neutron scattering work is supported by the U.S. Department of Energy (DOE) under EPSCoR Grant No. DE-SC0012432 with additional support from the Louisiana Board of Regents. Access to the neutron spin echo spectrometer and small-angle scattering instruments was provided by the Center for High Resolution Neutron Scattering, a partnership between the National Institute of Standards and Technology and the National Science Foundation under Agreement No. DMR1508249. Research conducted at the Spallation Neutron Source (SNS) at Oak Ridge National Laboratory (ORNL) was sponsored by the Scientific User Facilities Division, Office of Basic Energy Sciences, U.S. DOE.

\section{References:}

1. Taylor, T. M.; Davidson, P. M.; Bruce, B. D.; Weiss, J. Liposomal nanocapsules in food science and agriculture. Crit Rev Food Sci Nutr 2005, 45 (7-8), 587-605.

2. Woodka, A. C.; Butler, P. D.; Porcar, L.; Farago, B.; Nagao, M. Lipid bilayers and membrane dynamics: insight into thickness fluctuations. Phys Rev Lett 2012, 109 (5), 058102.

3. Nickels, J. D.; Cheng, X.; Mostofian, B.; Stanley, C.; Lindner, B.; Heberle, F. A.; Perticaroli, S.; Feygenson, M.; Egami, T.; Standaert, R. F.; Smith, J. C.; Myles, D. A.; Ohl, M.; Katsaras, J. Mechanical Properties of Nanoscopic Lipid Domains. J Am Chem Soc 2015, 137 (50), 15772-80.

4. $\quad$ Yi, X.; Shi, X.; Gao, H. Cellular uptake of elastic nanoparticles. Phys Rev Lett 2011, 107 (9), 098101.

5. Harland, C. W.; Bradley, M. J.; Parthasarathy, R. Phospholipid bilayers are viscoelastic. Proc Natl Acad Sci U S A 2010, 107 (45), 19146-50.

6. $\quad$ Simons, K.; Ikonen, E. Functional rafts in cell membranes. Nature 1997, 387 (6633), 569-72.

7. Weigel, A. V.; Simon, B.; Tamkun, M. M.; Krapf, D. Ergodic and nonergodic processes coexist in the plasma membrane as observed by single-molecule tracking. Proc Natl Acad Sci U S A 2011, 108 (16), 6438-43.

8. $\quad$ Seisenberger, G.; Ried, M. U.; Endress, T.; Buning, H.; Hallek, M.; Brauchle, C. Real-time single-molecule imaging of the infection pathway of an adeno-associated virus. Science 2001, 294 (5548), 1929-32.

9. He, Y.; Burov, S.; Metzler, R.; Barkai, E. Random time-scale invariant diffusion and transport coefficients. Phys Rev Lett 2008, 101 (5), 058101.

10. Szymanski, J.; Weiss, M. Elucidating the origin of anomalous diffusion in crowded fluids. Phys Rev Lett 2009, 103 (3), 038102.

11. Montero Llopis, P.; Jackson, A. F.; Sliusarenko, O.; Surovtsev, I.; Heinritz, J.; Emonet, T.; Jacobs-Wagner, C. Spatial organization of the flow of genetic information in bacteria. Nature 2010, 466 (7302), 77-81.

12. Kolesov, G.; Wunderlich, Z.; Laikova, O. N.; Gelfand, M. S.; Mirny, L. A. How gene order is influenced by the biophysics of transcription regulation. Proc Natl Acad Sci U S A 2007, 104 (35), 13948-53.

13. Hoffmann, I.; Michel, R.; Sharp, M.; Holderer, O.; Appavou, M. S.; Polzer, F.; Farago, B.; Gradzielski, M. Softening of phospholipid membranes by the adhesion of silica nanoparticles--as seen by neutron spin-echo (NSE). Nanoscale 2014, 6 (12), 6945-52.

14. Zilman, A. G.; Granek, R. Undulations and Dynamic Structure Factor of Membranes. Phys Rev Lett 1996, 77 (23), 4788-4791.

15. Watson, M. C.; Brown, F. L. Interpreting membrane scattering experiments at the mesoscale: the contribution of dissipation within the bilayer. Biophys J 2010, 98 (6), L9-L11.

16. Nagao, M.; Kelley, E. G.; Ashkar, R.; Bradbury, R.; Butler, P. D. Probing Elastic and Viscous Properties of Phospholipid Bilayers Using Neutron Spin Echo Spectroscopy. J Phys Chem Lett 2017, 4679-4684. 
17. Takeda, T.; Kawabata, Y.; Seto, H.; Komura, S.; Ghosh, S. K.; Nagao, M.; Okuhara, D. Neutron spin-echo investigations of membrane undulations in complex fluids involving amphiphiles. Journal of Physics and Chemistry of Solids 1999, 60 (8-9), 1375-1377.

18. Mell, M.; Moleiro, L. H.; Hertle, Y.; Fouquet, P.; Schweins, R.; Lopez-Montero, I.; Hellweg, T.; Monroy, F. Bending stiffness of biological membranes: what can be measured by neutron spin echo? Eur Phys J E Soft Matter 2013, $36(7), 75$.

19. Pencer, J.; Nieh, M. P.; Harroun, T. A.; Krueger, S.; Adams, C.; Katsaras, J. Bilayer thickness and thermal response of dimyristoylphosphatidylcholine unilamellar vesicles containing cholesterol, ergosterol and lanosterol: a small-angle neutron scattering study. Biochim Biophys Acta 2005, 1720 (1-2), 84-91.

20. Kucerka, N.; Tristram-Nagle, S.; Nagle, J. F. Structure of fully hydrated fluid phase lipid bilayers with monounsaturated chains. J Membr Biol 2005, 208 (3), 193-202.

21. Tristram-Nagle, S.; Nagle, J. F. HIV-1 fusion peptide decreases bending energy and promotes curved fusion intermediates. Biophys J 2007, 93 (6), 2048-55.

22. Lee, C. H.; Lin, W. C.; Wang, J. All-optical measurements of the bending rigidity of lipid-vesicle membranes across structural phase transitions. Phys Rev E Stat Nonlin Soft Matter Phys 2001, 64 (2 Pt 1), 020901.

23. Lee, J. H.; Choi, S. M.; Doe, C.; Faraone, A.; Pincus, P. A.; Kline, S. R. Thermal fluctuation and elasticity of lipid vesicles interacting with pore-forming peptides. Phys Rev Lett 2010, 105 (3), 038101.

24. Komura, S.; Takeda, T.; Kawabata, Y.; Ghosh, S. K.; Seto, H.; Nagao, M. Dynamical fluctuation of the mesoscopic structure in ternary C12E5-water-n-octane amphiphilic system. Phys Rev E Stat Nonlin Soft Matter Phys 2001, 63 (4 Pt 1), 041402.

25. Odeh, F.; Heldt, N.; Gauger, M.; Slack, G.; Li, Y. PFG-NMR Investigation of Liposome Systems Containing Hydrotrope. Journal of Dispersion Science and Technology 2006, 27 (5), 665-669.

26. Schneider, G. J.; Nusser, K.; Neueder, S.; Brodeck, M.; Willner, L.; Farago, B.; Holderer, O.; Briels, W. J.; Richter, D. Anomalous chain diffusion in unentangled model polymer nanocomposites. Soft Matter 2013, 9 (16), 4336.

27. Gerstl, C.; Schneider, G. J.; Fuxman, A.; Zamponi, M.; Frick, B.; Seydel, T.; Koza, M.; Genix, A. C.; Allgaier, J.; Richter, D.; Colmenero, J.; Arbe, A. Quasielastic Neutron Scattering Study on the Dynamics of Poly(alkylene oxide)s. Macromolecules 2012, 45 (10), 4394-4405.

28. Akimoto, T.; Yamamoto, E.; Yasuoka, K.; Hirano, Y.; Yasui, M. Non-Gaussian fluctuations resulting from power-law trapping in a lipid bilayer. Phys Rev Lett 2011, 107 (17), 178103.

29. Jeon, J.-H.; Javanainen, M.; Martinez-Seara, H.; Metzler, R.; Vattulainen, I. Protein Crowding in Lipid Bilayers Gives Rise to Non-Gaussian Anomalous Lateral Diffusion of Phospholipids and Proteins. Physical Review $X$ 2016, 6 (2).

30. Sharma, V. K.; Mamontov, E.; Ohl, M.; Tyagi, M. Incorporation of aspirin modulates the dynamical and phase behavior of the phospholipid membrane. Phys Chem Chem Phys 2017, 19 (3), 2514-2524.

31. Flenner, E.; Das, J.; Rheinstadter, M. C.; Kosztin, I. Subdiffusion and lateral diffusion coefficient of lipid atoms and molecules in phospholipid bilayers. Phys Rev E Stat Nonlin Soft Matter Phys 2009, 79 (1 Pt 1), 011907.

32. $\quad$ Pandey, R. B.; Anderson, K. L.; Farmer, B. L. Multiscale mode dynamics of a tethered membrane. Phys Rev E Stat Nonlin Soft Matter Phys 2007, 75 (6 Pt 1), 061913.

33. Mangiapia, G.; Gvaramia, M.; Kuhrts, L.; Teixeira, J.; Koutsioubas, A.; Soltwedel, O.; Frielinghaus, H. Effect of benzocaine and propranolol on phospholipid-based bilayers. Phys Chem Chem Phys 2017, 19 (47), 3205732071 .

34. Katsaras, J.; Gutberlet, T. Lipid Bilayers: Structure and Interactions. Springer-Verlag: Berlin, Heidelberg,, 2001. 